Documents pour l'histoire du français langue étrangère ou seconde

$24 \mid 1999$

Les auteurs classiques français dans l'enseignement du F.L.E. (18e et 19e siècles)

\title{
Les auteurs français et l'enseignement du F.L.E.
}

Quatre questions pour Avila

André Reboullet

\section{(2) OpenEdition \\ Journals}

Édition électronique

URL : https://journals.openedition.org/dhfles/3004

DOI : $10.4000 /$ dhfles.3004

ISSN : 2221-4038

Éditeur

Société Internationale pour l'Histoire du Français Langue Étrangère ou Seconde

Édition imprimée

Date de publication : 1 décembre 1999

Pagination : $9-22$

ISSN : 0992-7654

Référence électronique

André Reboullet, " Les auteurs français et l'enseignement du F.L.E. », Documents pour l'histoire du français langue étrangère ou seconde [En ligne], 24 | 1999, mis en ligne le 23 novembre 2014, consulté le 27 mai 2021. URL : http://journals.openedition.org/dhfles/3004; DOI : https://doi.org/10.4000/dhfles. 3004

Ce document a été généré automatiquement le 27 mai 2021.

(c) SIHFLES 


\title{
Les auteurs français et l'enseignement du F.L.E.
}

\author{
Quatre questions pour Avila
}

\author{
André Reboullet
}

1 Antoine Prost, un des meilleurs spécialistes français de l'histoire de l'enseignement, a rassemblé l'essentiel d'un cours donné en Sorbonne dans un ouvrage intitulé

2 Douze leçons sur l'histoire

3 Dans la quatrième leçon, les Questions de l'histoire, Prost, d'entrée, se demande: «Qu'estce qu'une question historique?», et donne cette réponse qui peut surprendre:

Il n'y pas de faits historiques par nature, le champ des objets potentiellement historiques est illimité... C'est la question qui va construire l'objet historique en procédant à un découpage original dans l'univers sans limite des faits et des documents possibles.

4 Et Prost conclut : «En un certain sens, une histoire vaut ce que vaut sa question».

5 C'est sous ce parrainage de Prost que nous placerons notre propos intitulé: Quatre questions pour Avila. Questions très générales, mais simples, elles tiennent en quatre mots: Quand? Quoi? Pourquoi? Qui?»

6 Leur généralité est telle qu'il est hors de propos d'y apporter des réponses nourries, sauf, parfois à titre d'exemple. Notre but, plus modeste et pourtant ambitieux, sera de cerner une problématique. Une cinquième question restera présente à notre esprit: avons-nous posé et proposé les bonnes questions? L'auditeur (ou le lecteur) en décidera.

7 Quand? Quoi? Pourquoi? Qui?, ces questions, nous les appliquerons à un domaine très précisément circonscrit: celui de la place de la littérature française et de son étude dans renseignement/apprentissage du français langue étrangère (ou FLE).

8 Nous n'ignorons pas que cet enseignement n'est pas un isolât et que, sauf pour les nécessités de notre analyse, il ne saurait être séparé d'autres composantes culturelles, par exemple, l'édition et la diffusion du livre et de la presse, les activités théâtrales ou les débats au sein des Académies. Ferdinand Brunot et Louis Réau y ont été très 
attentifs et en ont joué avec brio pour soutenir leur argumentation. Nous avons choisi une voie étroite et moins brillante.

Plus encore, nous avons poussé notre volonté de dépouillement jusqu'à ne choisir, dans l'enseignement/apprentissage du français que les premières périodes, les plus difficiles, les plus pédestres.

On le devine, nos objectifs son étroitement didactiques; notre propos s'inscrit dans une histoire de pédagogie concrète. Ou primaire, si l'on veut.

11 Et pour mieux me situer dans le terre à terre, je me permettrai une seconde citation que j'emprunte à Marie-Barbe Leprince de Beaumont, l'auteur du Magazin des Enfans.

Je me suis convaincu absolument d'une chose que je soupçonnais. Le dégoût des enfants pour la lecture vient de la nature des livres qu'on leur met entre les mains; ils ne les comprennent pas et, de là, naît inévitablement l'ennui».

\section{Quand?}

12 Tout nous invite à commencer par la question quand? Et d'abord l'intitulé du programme d'Avila qui cerne deux périodes, le $17^{\mathrm{e}}$ et le $18^{\mathrm{e}}$ siècles pour la création des oeuvres de l'âge classique, le $18^{\mathrm{e}}$ et le $19^{\mathrm{e}}$ pour leur réception, au total trois siècles.

Mais, comme tout historien le sait, travailler sur une période limitée, c'est obligatoirement, et sans qu'on y mette malice, commencer par fouiner en deçà et prolonger au-delà. Ainsi, notre quand trop simple devra-t-il se décomposer en deux sous-questions: à partir de quand? jusqu 'à quand?

$C^{\prime}$ est au $18^{\mathrm{e}}$ siècle, au $18^{\mathrm{e}}$ siècle d'abord, que le texte littéraire va faire une entrée remarquée dans l'enseignement du FLE. Et le mouvement commencé va se poursuivre et s'accentuer au $19^{\mathrm{e}}$ et au $20^{\mathrm{e}}$ pour des raisons différentes (nous y reviendrons).

Mais ce serait une vision bien étroitement manichéenne de penser que si le texte littéraire ne trouve sa place qu'à partir du $18^{\mathrm{e}}$, les deux siècles qui l'ont précédé, $16^{\mathrm{e}}$ et $17^{\mathrm{e}}$, vivaient une période d'ignorance où les belles lettres n'avaient que faire dans un enseignement pragmatique de la langue française.

Et se pose alors la question: À partir de quand?

17 Sans remonter jusqu'au Moyen-âge, on aura, dès le $16^{\mathrm{e}}$ siècle, quelques prises et bonnes surprises qui invitent à réfléchir. Que Gabriel Meurier, un maître de langues anversois, dans une compilation intitulée Le Thrésor des sentences dorées, proverbes et dicts communs, etc., publié en 1568, s'autorise deux citations littéraires, l'une de Clément Marot, l'autre d'un grand rhétoriqueur, Jean Molinet, c'est sympathique, mais homéopathique... Une hirondelle ne fait pas le printemps.

Prêtons attention, plutôt, à trois maîtres de français, installés en Angleterre, toujours au $16^{\mathrm{e}}$ siècle: Claude Hollyband, Jacques Bellot et John Eliot.

Certes aucun ne reproduit dans ses oeuvres pédagogiques le moindre texte littéraire, mais tous trois invitent leur élève-lecteur à lire, au terme de ses études, une sélection très précise et, pour nous, très précieuse d'œuvres littéraires.

Hollyband y a recours par deux fois: en 1576, dans The French Littleton, il conseille la lecture de Philippe de Commines «dans une éditions corrigée» et les oeuvres de Pierre Boaystuau, auteur qu'il qualifie de «plus éloquent et meilleur écrivain de langue française»; plus tard, en 1588, dans De Pronontiatione Linguae Gallicae, Hollyband 
recommande l'Amadis de Gaule, les oeuvres de Clément Marot, le Printemps du poète Yver.

21 Jacques Bellot, contemporain d'Hollyband, veut que son élève puisse lire, lui aussi, l'Amadis (dans la version de Des Essarts) et Marot mais il ajoute Théodore de Beze, Froissait, Ronsard, Collet, Jodelle, Pibrac et Du Bartas.

John Eliot a choisi Boaystuau, Marot, Ronsard, Du Bartas, mais aussi Amyot et Philippe de Mornay.

Quelles conclusions tirer des ces trois sélections?

La première est que ces choix n'ont eu, probablement, aucune incidence - ou qu'une incidente médiocre sur l'enseignement donné par le maître, face à ses élèves. Donc, que la première solution dans la relation littérature et enseignement du FLE a été la moins courageuse: le renvoi à plus tard. Les sélections proposées étaient destinées à ce qu'un journaliste sportif appellerait aujourd'hui la «troisième mi-temps», un temps indéfini que l'élève, au terme de son apprentissage, pouvait utiliser à son gré pour tout faire ou ne rien faire.

Au-delà de ce constat, un peu court et désabusé, nous pourrons prolonger la réflexion.

$1^{\circ}$ ) en faisant remarquer qu'en proposant in fine une sélection littéraire, les trois maîtres ont pris en compte le littéraire, même s'ils l'ont évacué, rapidement, hors du champ scolaire;

$2^{\circ}$ ) en suggérant que cette prise en compte concourrait à valoriser leur enseignement et leurs ouvrages; car, à cette époque, les belles lettres n'étaient pas l'apanage des marchands, mais celui des nobles et des clercs. On peut penser qu'Hollyband, toujours soucieux de sa dignité docente, a été sensible à cet aspect des choses;

$283^{\circ}$ ) en reliant ces choix littéraire à une possible volonté des maîtres de langue de tenir, au-delà de leur métier stricto sensu un rôle d'intercesseur culturel à une époque où, grâce à l'imprimerie, la littérature française, en traduction surtout, commençait à devenir disponible pour un public de «lecteurs-scripteurs», évalué en Angleterre, à la fin du $16^{\mathrm{e}}$ siècle à 25 pour cent de la population.

En 1595, Hollyband traduit en anglais et publie le Théâtre du monde de Pierre Boaystuau. On peut s'interroger. Notre maitre n'a-t-il pas mis au point une stratégie en deux temps? D'abord appâter l'élève-lecteur par un éloge démesuré de Boaystuau dans le Littleton; ensuite l'inviter à une lecture réaliste, c'est-à-dire en traduction? Deuxième solution possible de la relation littérature/enseignement du FLE;

$4^{\circ}$ ) en mettant en relief le souci de contemporanéité dans ces trois sélections. A deux exceptions près, Froissait et Commines, les auteurs appartiennent au $16^{\mathrm{e}}$ siècle;

$315^{\circ}$ ) en faisant observer la prépondérance accordée à la poésie (sans doute en partie pour la brièveté de ses oeuvres, commode pour l'étude) et aux textes historiques qui connaîtront toujours la vogue dans les textes littéraires français proposés aux étrangers. Herbert Christ qui a remarqué cette sur-représentation de l'histoire au $19^{\mathrm{e}}$ siècle en Allemagne se demande si l'histoire n'avait pas, alors, la fonction qui est celle aujourd'hui des études de civilisation. Une hypothèse intéressante à vérifier...

$6^{\circ}$ ) en étant attentif au succès ou à l'insuccès des différents genres littéraires; ou de tel ou tel auteur. Il est aussi important de s'interroger sur la présence que sur l'absence des uns et des autres. Pourquoi Boaystuau, pourquoi Pibrac dont les Quatrains, mal 
rimaillés, nous semblent aujourd'hui d'une insupportable platitude? Pourquoi Marot et Ronsard, et pas Du Bellay? Pourquoi pas Rabelais? Ou Montaigne?

Nous pourrions mener sur le $17^{\mathrm{e}}$ siècle une réflexion comparable. Limitons-nous à quelques faits signifiants:

les premières publications à l'étranger d'œuvres littéraires françaises dont on peut admettre qu'elles ont pu être utilisées dans «l'après-scolaire».

Par exemple, en 1604, à Amsterdam, les Quatrains de Pibrac, déjà publiés en France en 1574:

- une des premières anthologies littéraires, un genre qui fera florès par la suite, celle de J. Corbinelli (Amsterdam, 1681) dont le titre est tout un programme:

Extrait de tous les beaux endroits des ouvrages des plus célèbres autheurs de ce temps

- la lecture en Angleterre, à la fin du $17^{e}$ siècle, des romans, de Mme de Scudery, Gomberville, La Calprenède, dont J.-C. Chevalier a pu dire: «La lecture de ces romans, c'est le sommet de l'enseignement de Claude Mauger».

Littérature, textes littéraires et enseignement du FLE; on peut déceler du $16^{\mathrm{e}}$ siècle à nos jours, avec des variantes importantes, une constante relation, une continuité où l'on distinguera des temps forts dus à des causalités, fortes elles-aussi. Nous en proposerons trois:

$\left.401^{\circ}\right)$ La naissance et le développement foudroyant de l'imprimerie entre 1450 et 1500 , sans laquelle l'entrée des textes littéraires et leur lecture à l'étranger n'auraient pas été possibles dès les débuts $\mathrm{du} 16^{\mathrm{e}}$ siècle. Il vaudrait la peine de rechercher les concordances entre les progrès de l'imprimerie en Allemagne, Angleterre, Espagne, Italie et ceux de la diffusion scolaire et, plus encore, extra-scolaire de la littérature française.

$412^{\circ}$ ) L'exceptionnel dynamisme culturel de la France au $18^{\mathrm{e}}$ siècle, même si ceux qui l'ont analysé et célébré, Réau ou Brunot, ont fait la part trop belle à cette expansion. On trouvera chez Robert Mandrou une vue plus juste de la réalité. L'équité impose d'équilibrer l'offre culturelle française par une demande européenne, expression de ce que Reinhard Wittmann appelle «la folie de la lecture», une maladie qui a gagné très vite l'Allemagne et l'Angleterre puis s'est propagée dans le Sud et l'Europe centrale.

$423^{\circ}$ ) Dans la deuxième moitié du $19^{\mathrm{e}}$ siècle, l'introduction systématique des langues modernes dans le cursus des études secondaires en Europe, plus rarement hors Europe.

Ici plus que le quantitatif, augmentation du nombre d'élèves ou du nombre d'heures d'enseignement, on devra prendre en compte:

44 - la volonté des «nouveaux» professeurs de langues modernes d'établir que les humanités modernes ont une valeur culturelle au moins égale à celle des humanités classiques. Et quel meilleur moyen de l'établir que par le truchement d'une littérature qui était, qu'on le veuille ou non, une des plus riches de l'humanité?

45 - le souci, plus discret, qu'ont eu une partie de ces «nouveaux enseignants», appelonsles les «récupérés», qui, il y a peu étaient d'humbles tâcherons courant le cachet de se démarquer de leur origine mercantile en faisant place à une culture littéraire nouvelle qui pouvait justifier leur promotion et leur nouveau statut. 


\section{Quoi?} que, dans le Magazin, Madame Bonne occupe le rôle de Mentor et qu'à Télémaque (le personnage) succède un essaim de jeunes Anglaises de 5 à 13 ans. Mais cette similitude ne doit pas cacher les disparités entre les deux oeuvres. Et pour expliquer le succès du Magazin les hypothèses de l'auteur restent les plus probables. Madame Leprince a voulu 
écrire dans un style plus simple que celui de Fénelon et elle y a réussi, tout en restant «amusante». Surtout elle a eu l'intelligence de viser un public très précis et très porteur: celui des jeunes lecteurs et surtout lectrices. En cela, Madame Leprince est moderne: elle a saisi l'intérêt de délimiter un public.

Avec le Magazin apparaît une troisième solution dans la relation littérature/ enseignement du français, celle de ce que l'on pourrait appeler l'ersatz littéraire. Du Magazin à nos modernes rewritings en français facile, une filière va se constituer.

Le $19^{\mathrm{e}}$ siècle, le siècle surabondant, nous l'envisagerons à partir de deux documents:

62 Le choix de livres, en quelque sorte le programme de lecture que, sous le titre Bibliothèque française, Nicolas Chantreau propose à ses lecteurs en fin d'ouvrage;

L'inventaire des programmes bavarois (Schulprogramme) de 1845 à 1913, inventaire établi par Herbert Christ dans Documents, $\mathrm{n}^{\circ} 6$.

La Bibliothèque française de Chantreau, telle qu'elle apparaît dans le célèbre Arte de hablar bien francés (Barcelone, édition de 1858) propose pour la seule rubrique littérature 20 auteurs différents (et 20 ouvrages) dont 5 pour le théâtre; à quoi s'ajoutent 4 théoriciens de la littérature, une dizaine d'historiens dont Michelet. Ces auteurs et ouvrages appartiennent majoritairement au $17^{\mathrm{e}}$ siècle, un au $18^{\mathrm{e}}$ (Voltaire) et deux au $19^{\mathrm{e}}$ (Chateaubriand et Michelet).

65 Cette abondance de noms est en quelque sorte multipliée par l'invitation faite à l'étudiant de lire des oeuvres complètes: ainsi, l'Histoire de France de Michelet en 5 volumes in $8^{\circ} \ldots$

D'une édition à l'autre, des variantes sont curieuses. Chantreau, édition de recommande les Contes moraux de Marmontel (3 volumes), l'Histoire de Gil Blas, 4 volumes, les oeuvres de Condillac ( 21 volumes). L'édition de 1805 y ajoute Fontenelle, 11 volumes. Mais en 1858, ces quatre auteurs du $18^{\mathrm{e}}$ et leurs oeuvres auront disparu. Ils ont peut-être cessé d'être à la mode?

Herbert Christ et la Bavière nous situent devant un autre aspect de la surabondance. Dans les shulprogrammes bavarois, on ne trouve pas moins de 203 auteurs différents, 550 titres d'ouvrage dont 19 anthologies de poésie, 14 titres de colletions de textes en prose, 13 titres de traités d'histoire littéraire. Parmi les auteurs les plus cités, Chateaubriand, Corneille, Daudet, Hugo, Lamartine, Molière, Racine, Vigny, Voltaire mais aussi, hélas!, François Coppée, Casimir Delavigne, Emile Souvestre, André Theuriet...

Contraste donc entre un $18^{\mathrm{e}}$ un peu maigrelet et un $19^{\mathrm{e}}$ pléthorique? À mieux y regarder, il convient de nuancer.

$\mathrm{Au} 18^{\mathrm{e}}$, le trop grand éclat de Télémaque ou du Magazin des enfans nous a caché quelques auteurs et ouvrages qui ont eu, dans l'enseignement du français, une carrière plus qu'honorable. Ainsi Marmontel avec ses Contes moraux ou Madame de Genlis, en fin de siècle, avec Adèle et Théodore ou Les veillées du Château. Une liste que l'on pourrait considérablement allonger avec les seuls travaux de deux historiens polonais du domaine: Anna Nikliborc et Lucjan Grobelak, lesquels font état, dans les lectures des jeunes Polonais, de La Fontaine, Corneille, Racine, Molière, Pascal, Beaumarchais, Berquin, Rollin et beaucoup d'autres.

70 Pour le $19^{e}$, on s'interroge. Quel impact réel ont pu avoir les listes foisonnantes, démentielles parfois, de Nicolas Chantreau sur les lectures d'élèves, compte tenu du 
temps et des moyens financiers dont ils pouvaient disposer? Les inventaires de Christ doivent être resitués dans une période qui a duré 68 ans et dans un état important, la Bavière, qui devait compter un nombre conséquent d'établissements.

Plus que ce contraste relatif, on devrait prendre attention à deux phénomènes plus significatifs:

72 Le succès durable d'un genre littéraire, le conte, déjà présent avec Madame Leprince de Beaumont (le Magazin contient 16 contes, qui représentent plus de $40 \%$ de l'ouvrage), avec Madame de Genlis ou Marmontel mais qui ne cessera ensuite d'avoir la faveur du public scolaire, avec André Théuriet, Jules Claretie, Emile Souvestre et, très au-dessus, Alphonse Daudet et Guy de Maupassant. La Ficelle, le Petit Fût et La Parure ont fait le tour du monde et des générations de lycéens, sur les cinq continents, connaissent mieux la Normandie de Maupassant que le Paris des Misérables ou de Zola. Un succès qu'explique la caractéristique principale du genre: la brièveté d'un texte formant une unité de sens.

En contrepoint et complément une formule nouvelle va se propager: l'anthologie ou recueil de morceaux choisis. Elle a, comme le conte, l'avantage de la brièveté des textes, même si ceux-ci, à la différence du conte, sont un peu «haut le pied». Le florilège, en revanche, a l'avantage d'offrir une ouverture sur la diversité du littéraire.

\section{Pourquoi?}

Pour traiter de notre troisième question: les textes littéraires, pourquoi?, nous ne disposons que de peu de matière.

Pour faire bonne mesure, prenons le plus bavard de nos auteurs. Dans les 30 pages de l'Avertissement du Magazin des enfans, l'immanquable Madame Leprince nous donne un témoignage attachant sur sa double et triste condition de maîtresse de français et d'auteur mais s'en tient sur les finalités de son ouvrage et de son enseignement aux banalités les plus convenues: avant tout, «former les mœurs», "ouvrir l'esprit», se servir sans les séparer et, moins encore, les opposer «de la religion et de la raison»...

Plutôt que d'inventorier de tels témoignages qui risqueraient d'être souvent récurrents, il nous a paru plus opératoire, quitte à frôler l'anachronisme, d'utiliser une opposition moderne et de distinguer, dans l'utilisation des textes littéraires, ce qui relève des moyens et ce qui relève des finalités.

77 Le texte littéraire, dans l'esprit de ceux qui l'emploie, remplit une double fonction: ludique et d'enrichissement linguistique.

78 Ludique. Mme Leprince de Beaumont, encore elle, le souligne sans ambages. Elle a soumis, avant publication, son manuscrit à des «personnes faites» et à ses jeunes écolières. Les unes et les autres lui disent la même chose: «elles se sont amusées à la lecture du manuscrit». Et cet amusement décide l'auteur à publier. Même si cette explication sent son artifice et son mercantilisme, il faut en croire l'auteur. Le vieil adage «il faut savoir instruire et plaire» est toujours valable et les enseignants comme les auteurs ont désormais compris que les textes littéraires -ou pseudo-littérairespouvaient être un excellent dérivatif à des dialogues dix fois répétés et un peu lourdement terre à terre.

79 Enrichissement linguistique. Là encore, risquons une notion moderne: celle de texte authentique. 
Pour l'essentiel, depuis les origines, les outils d'enseignement du français sont écrits en «français fabriqué». L'écolier ne connaît, s'il est anglais, que la prose de Monsieur Hollyband ou de Monsieur Bellot, ou celle de Monsieur Claude Mauger, s'il vit au $18^{\mathrm{e}}$. À deux exceptions près: dans The french Littleton (mais aussi dans d'autres ouvrages comparables) il pourra lire de longues listes de proverbes français et des textes religieux bien connus, tels que le Notre Père, le Credo, le $5^{\mathrm{e}}$ chapitre des Actes des Apôtres, une Oraison... C'est la part, très congrue, de l'authentique.

Un nouvel authentique, plus riche, plus fonctionnel, apparaît avec le texte littéraire. Il sera outil d'enrichissement linguistique parce qu'il propose à l'élève des modèles lexicaux et syntaxiques nouveaux; il sera aussi outil d'évaluation linguistique parce qu'il ne se borne pas à la seule forme dialoguée et souvent catéchistique des colloques et que, pour l'aborder et le comprendre, pour le lire et le traduire (on ne fera jamais autre chose), l'étudiant sera à même déjuger sa véritable compétence.

Les finalités de l'étude littéraire ont eu, selon l'époque, une importance variable. On a $\mathrm{vu}$, avec Madame Leprince, combien la finalité morale a pu être primordiale sous le double aspect de la formation de l'esprit et celle de l'âme. Ou du cœur. Oserons-nous dire, oui nous l'osons, que cette primauté va, tout au long du $19^{\mathrm{e}}$, puis du $20^{\mathrm{e}} \mathrm{s}^{\prime}$ estomper progressivement, sans toutefois jamais complètement disparaître.

3 La finalité esthétique a moins subi l'érosion du temps. Que la langue française, par le truchement de sa littérature propose des modèles linguistiques uniques, toujours renouvelés dans leur diversité, ce que Marc Fumaroli, après beaucoup d'autres dont Rivarol, appelle «le génie de la langue française», est aujourd'hui encore un sujet de débat où partisans et adversaires du fameux «génie» discutent avec âpreté. Mais, pour notre propos, le débat contemporain ne nous importe pas. Il suffit pour notre analyse, de constater que la finalité esthétique a toujours été présente, aux deux derniers siècles, dans l'argumentaire en faveur du français, en général, et de la littérature, en particulier. Une seule restriction: à trop se fixer sur le style, sur les qualités de langue, on a souvent, très souvent, manqué ce qui fait la spécificité du littéraire. Nouveau débat, dans lequel nous n'entrerons pas.

Que la littérature française ait une finalité sociale, qu'elle ait proposé àceux qui l'étudient des modèles sociaux et, au-delà, un modèle national est à la fois évident et flou. Flou parce qu'apparait ici, à ce niveau de notre analyse, le danger des limites très, trop étroites, que nous nous sommes fixés: le seul cadre de l'enseignement du français. A l'évidence, Brunot ou Réau qui ne séparent pas l'enseignement des autres facteurs culturels, sont plus à l'aise pour traiter ce que nous ne ferons pas, de la gallomanie ou des afrancesados.

Nous venons d'affirmer aussi que la littérature française proposait un modèle national. Qui mieux qu'un étranger, l'Allemand Ernst-Robert Curtius, dans son Essai sur la France, a su caractériser la spécificité de ce modèle?

Écoutons-le:

La littérature joue un rôle capital dans la conscience que la France prend d'ellemême et de sa civilisation. Aucune autre nation ne lui accorde une place comparable. Il n'y a que la France où la nation entière considère la littérature comme l'expression représentative de ses destinées.

Avec Curtius, nous avons changé de domaine. Nous parlions jusqu'ici de littérature. Nous avons abordé la civilisation... 


\section{Qui?}

établit comment la floraison des études littéraires dans l'enseignement secondaire s'explique en amont par la place privilégiée que tenait la littérature, française en la circonstance, dans la formation universitaire des professeurs. Et ce qui est vrai pour la Bavière l'est ou le sera pour beaucoup de pays en Europe et hors d'Europe. On se doit de rendre hommage à l'excellent niveau des universitaires francisants dès la fin du $19^{\mathrm{e}}$ siècle et à la qualité des professeurs qu'ils ont formés. Ainsi du Brésilien Mario Candido, excellent historien et théoricien de la littérature qui illustre la qualité des études françaises à l'Université de Sâo Paulo et justifie la part que les Français ont pris à la création de la Faculté de Philosophie. notre Quai d'Orsay, on a appelé les missionnaires (ou les détachés), ces milliers de professeurs français et dont la formation littéraire est d'excellente qualité, de meilleure qualité, souvent, que la formation pédagogique ou linguistique. les plus brillants.

6 On connaît quelques phares illustres: Voltaire en Prusse, Diderot en Russie, Beaumarchais, Montesquieu, Voltaire en Angleterre; sans oublier Chateaubriand qui fut, incognito, maître de français en quelques sorte par inadvertance. Avec raison, Robert Mandrou fait aussi un sort aux écrivains du second rang, aujourd'hui inconnus, oubliés mais qui, je cite Mandrou «n'en jouent pas moins un rôle essentiel de diffuseur sans prestige qui diluent, explicitent, voire déforment les idées forces qui se discutent 
dans les sociétés savantes et dans les salons français». Et, en exemple, Mandrou cite l'abbé Delille qui séjourna en Turquie, en Suisse, en Allemagne et en Angleterre.

Les diplomates, enfin, les diplomates -écrivains surtout- ont été des acteurs efficaces.

Depuis longtemps: Du Bellay au $16^{\mathrm{e}}$ siècle, et à Rome; le Cardinal de Bernis au $18^{\mathrm{e}}$. A une époque plus récente, Claudel, Giraudoux, Morand, de Bourbon-Busset qui, tous, ont occupé des postes diplomatiques.

E.-R. Curtius a pu écrire: «C'est au Ministère des Affaires étrangères, au Quai d'Orsay, qu'ont été écrits quelques-uns des livres les plus marquants de la littérature française d'après-guerre». Précisons: d'après la guerre de 1914-1918.

Trois remarques pour non pas conclure, mais seulement terminer:

L'histoire des relations entre français langue étrangère et littérature est celle d'une série d'oppositions, de contradictions, de conflits et d'une série d'essais plus ou moins heureux, jamais définitifs, de solutions et de compromis, de réussites et de coups de cœur, pour un auteur ou un livre.

littérature souhaitée... et... littérature repoussée dans l'après-scolaire pratiques lourdement pédagogiques et finalités prestigieuses humbles vulgarisateurs et propagandistes illustres littérature glorieuse versus littérature studieuse.

Aujourd'hui, comme hier, les professeurs en exercice doivent être attentifs à trois dominantes, mais qui peuvent être dérivations et pièges: la tentation de la brièveté. Bien sûr, et même au $18^{\mathrm{e}}$ siècle, on n'a jamais lu Télémaque ou le Magazin en entier, dans les classes. Au mieux on en lisait le quart ou le dixième en une année scolaire.

N'en concluons pas qu'il faudrait se limiter aux contes, aux fables, à un sketch de Raymond Devos, au théâtre de Jean Tardieu ou à quelques maximes de La Roche Foucault. Et cherchons des formules qui concilient lecture partielle et étude d'ensemble de grands et longs textes.

4 La tentation de la simplicité des textes. Aujourd'hui on choisit volontiers les poèmes de Prévert, Vol de nuit, l'Étranger, le dernier Le Clézio parce que ses oeuvres ont la réputation d'être simples. Voilà, pourtant, trente ans que Daniel Coste a montré que l'Étranger n'avait qu'une apparence de simplicité et qu'en le méconnaissant on risquait le contre-sens. Les vraies oeuvres littéraires, hélas, sont complexes.

5 Latentation de la contemporanéité. Tous les étudiants dans le vaste monde veulent lire des modernes. Pourquoi pas? Qui ne se réjouit d'observer que les études sur les littératures francophones: africaine, antillaise, québécoise, toutes récentes, sont, hors de France, notamment en Allemagne, d'une qualité comparable aux études menées en France? À ce détail près que tout moderne est aussi un héritier. Et que cet héritage ne peut pas être sans danger, méconnu ou gommé.

J'ai largement débordé les cadres, pourtant larges, fixés pour Avila. Pardon!

7 J'ai toujours pensé qu'Avila devrait être le premier d'une série de colloques sur le littéraire. Par exemple sur la place de la littérature espagnole dans l'étude de la langue espagnole; ou de la littérature anglaise dans l'étude de l'anglais.

Dans cette future galaxie des colloques littéraires, Avila c'est l'Étoile du Berger, la première à apparaître dans le ciel, la dernière à s'éteindre.

Merci de m'avoir accordé une place dans cette Étoile! 


\section{AUTEUR}

\section{ANDRÉ REBOULLET}

Le Français dans le Monde 